\title{
Métodos para aplicar a Gamificação no Design de Serviços: pelo viés da Gestão de Design e Abordagem Sistêmica
}

Methodologies to apply the gamification in the design of services: by the bias of design management and systemic approach

FIGUEIREDO, Luiz Fernando Gonçalves de; Pós-Doutor; UFSC - NAS Design

Iffigueiredo2009@gmail.com

FERREIRA, Alais Souza; Mestranda em Design; UFSC - NAS Design

alais.ferreira@live.com

MACEDO, Thales Eduardo; Graduado; Universidade Federal de Santa Catarina - Animaking

thales@animaking.com.br

\section{Resumo}

No design, a gamificação pode auxiliar na simplificação do trabalho ou no processo de aprendizagem durante a busca por soluções; está em crescente aceitação como método de resolução de problemas; e pode melhorar o envolvimento dos usuários. Assim, objetiva-se identificar metodologias que auxiliem a aplicação da gamificação no processo de Design de Serviço (DS) pelo viés da Gestão de Design (GD) e Abordagem Sistêmica (AS). A pesquisa classifica-se como teórica, quantitativa, qualitativa e exploratória, constituída pela junção de duas revisões sistemática da literatura (RSL's). Como resultado de pequisa, conclui-se que a gamificação pode potencializar a motivação e o engajamento (que podem ajudar na resolução de problemas no DS e GD). Ressalta-se, ainda, que a gamificação pode ser aplicada no DS por meio de metodologias gamificadas, como: Métrica de Engajamento; Modelo de Gamificação Canvas; Game On! Toolkit; Octalysis; Caleidoscópio de gamificação eficaz; Método de aplicação da gamificação; Modelo de mecânica da gamificação; Ciclo de gamificação; IdeaChef; e Gamification Service Framework.

Palavras Chave: gestão de design, abordagem sistêmica, design de serviços, gamificação, metodologia.

\begin{abstract}
In design, gamification can assist in simplifying the work or learning process during the search for solutions; It is in increasing acceptance as a method of problem solving; And it can improve user involvement. Thus, it aims to identify methodologies that assist the application of gamification in the process of service design (SD) by the bias of Design management (DM) and systemic approach (SA). The research is classified as theoretical, quantitative, qualitative and exploratory, consisting of the junction of two systematic reviews of the literature (RSL's). As a result of research, it is concluded that gamification can enhance motivation and engagement (which can help solve problems in SD and DM). It is also emphasized that gamification can be applied to the SD by means of gamificadas methodologies, such as: Métrica de Engajamento; Modelo de Gamificação Canvas; Game On! Toolkit; Octalysis; Caleidoscópio de gamificação eficaz; Método de aplicação da gamificação; Modelo de mecânica da gamificação; Ciclo de gamificação; IdeaChef; and Gamification Service Framework.
\end{abstract}

Keywords: design management, systemic approach, service design, gamification, methodology. 


\section{Introdução}

Desde os tempos remotos os mecanismos dos jogos estão presentes na forma de viver e se relacionar do ser humano, por isso, não se pode entender a gamificação como algo socialmente novo. O termo gamificação foi criado entre 2002 e 2003 por Nick Pelling, ganhando popularidade oito anos depois quando McGonigal (2012) realizou uma apresentação de TED. No início do século $X X$, devido ao surgimento da gamificação, o jogo deixou de ser considerado uma atividade destinada à distração e passou a ser utilizado pelos negócios como uma forma de adquirir conhecimento, desenvolvimento pessoal e profissional. Dessa forma, a gamificação surgiu como resposta às necessidades do indivíduo e da sociedade pós-moderna.

A gamificação deriva diretamente da popularização dos jogos e de suas capacidades intrínsecas e extrínsecas de motivar a ação, resolver problemas e potencializar aprendizagens lógicas e/ou criativas nas mais diversas áreas do conhecimento (FORMANSKI, 2016). Na área de design, a gamificação pode auxiliar na simplificação de um trabalho ou no próprio processo de aprendizagem durante a procura por soluções. Olenski (2014) informa que essa abordagem gamificada está encontrando crescente aceitação como um método de resolução de problemas e pode ser utilizada para melhorar o envolvimento dos usuários e de outros importantes indicadores de coleta de dados.

Diante do exposto, questiona-se: Como aplicar a gamificação no design de serviço? Já existem métodos próprios para isso? Assim, objetiva-se identificar métodos que auxiliem a aplicação da gamificação no processo de Design de Serviço pelo viés da Gestão de Design e Abordagem sistêmica. Para isso foi necessário primeiramente agrupar as pesquisas, relacionadas ao assunto abordado, coletadas em duas Revisões Sistemáticas da Literatura (RSL); no segundo momento verificar se possuía pesquisas relevantes nas referências bibliográficas do portfólio final dos trabalhos selecionados nas RSL's; e na sequência relatar os métodos de gamificação aplicados por prestadores de serviços que implementaram a gamificação. Esta pesquisa classifica-se por sua natureza como básica e teórica, pela abordagem como quantitativa e qualitativa, pelos objetivos como exploratória. De acordo com os procedimentos metodológicos, o estudo é classificado como RSL e levantamento bibliográfico de dados secundários.

De acordo com Patrício (2016), tanto um método quanto uma ferramenta gamificados podem influenciar positivamente o "bom" comportamento dos funcionários no local de trabalho, uma vez que: desencadeia a curiosidade das pessoas pela inovação; mantém as pessoas intrinsecamente motivadas para se envolver continuamente; aprimora a colaboração interna; promove maior diversão e compromisso entre os funcionários; aumenta a motivação para aprender e crescer; fornece ideias para futuras áreas de aplicações de produtos ou serviços; aumenta a vontade de tomar riscos; educa sobre como aceitar o fracasso; promove a abertura a novas ideias e tecnologias; entre muitos outros comportamentos. Isto justifica a relevância desta pesquisa e contribui para o campo teórico bem como para o prático, pois auxilia na identificação desses métodos gamificados direcionados para o Design de Serviço, incentivando a reflexão sobre o assunto.

\section{Gestão de Design}

O gerenciamento por meio de estratégias planejadas, métodos, técnicas e ferramentas do Design podem oferecer vantagens competitivas a uma empresa no mercado. Nesse ambiente, a gestão necessariamente insere-se de modo estratégico em todo o processo e níveis: operacional, 
tático e estratégico (AGUIAR et al., 2015). O processo de Gestão de Design (GD) demanda a participação e o comprometimento de todos os membros da organização, incluindo o usuário (KRUCKEN, 2009). Dessa forma, o Designer é o gestor que irá articular essas comunicações, facilitando e apoiando o desenvolvimento de inovações no processo de criação de produtos (tangíveis) e serviços (intangíveis).

Assim, a GD consiste em uma atividade articuladora e multidisciplinar com abordagem holística que promove "o gerenciamento bem-sucedido de pessoas, projetos, processos e procedimentos que estão por trás da criação dos produtos, serviços, ambientes e experiências que fazem parte de nossa vida diária" (BEST, 2012, p.8). Segundo Mozota (2011), busca implementar o planejamento de design em uma empresa, afim de ajudá-la a alcançar seus objetivos e melhorar a qualidade de seus produtos, serviços e equipes.

Nesse sentido, o design busca por ferramentas específicas, para formulação estratégica, gestão de ideias, modelos e protótipos para inovação, bem como ferramentas de comunicação para ampliar fronteiras (MOZOTA, 2011). Dessa forma, na prática, a GD controla a administração do valor agregado do design, dos relacionamentos e dos processos, podendo auxiliar na identificação dessas ferramentas. A próxima seção relata a abordagem holística denominada como Abordagem Sistêmica do Design.

\section{Abordagem Sistêmica do Design}

A abordagem sistêmica (AS) transfere o foco dos objetos para o estudo holístico dos sistemas envolvendo todos os seus processos e suas interdependências, uma vez que cada um dos elementos ao serem reunidos para construir uma unidade funcional maior, desenvolvem qualidades que não se encontram em seus componentes isolados (BERTALANFFY, 2014). Em vez de essências e de substâncias, a organização; em vez das unidades simples e elementares, as unidades complexas; em vez dos agregados formando corpos, os sistemas de sistemas de sistemas (MORIN, 2005).

Essa visão é considerada holística por conceber o mundo como um todo integrado. Segundo Capra (2010), a ênfase nas partes tem sido chamada de mecanicista ou reducionista; a ênfase no todo, de holística, organísmica ou sistêmica. O seu principal desafio é desenvolver e suportar o desenvolvimento de soluções a questões de alta complexidade, a fim de mudar as formas de pensar nos projetos por meio de uma visão mais ampla, envolvendo produtos, serviços e comunicação de forma conjunta e sustentável.

A AS presente no processo de design mostra como deve acontecer o trabalho dentro do processo de criação do objeto (produto ou serviço) para que o mesmo seja aperfeiçoado, entendendo que cada parte possui sua importância e deve estar presente dentro de um sistema maior. Santos (2000, p. 23) propõe uma abordagem na qual o design é considerado um sistema processador de informações. Vasconcellos (2013) e Bertalanffy (2014) complementam ao informarem que o sistema é um conjunto de elementos (ou subsistemas) que interagem entre si, com objetivo comum e que evoluem com o tempo (Figura 1). 
Figura 1 - Elementos do sistema de Design como processador de informações
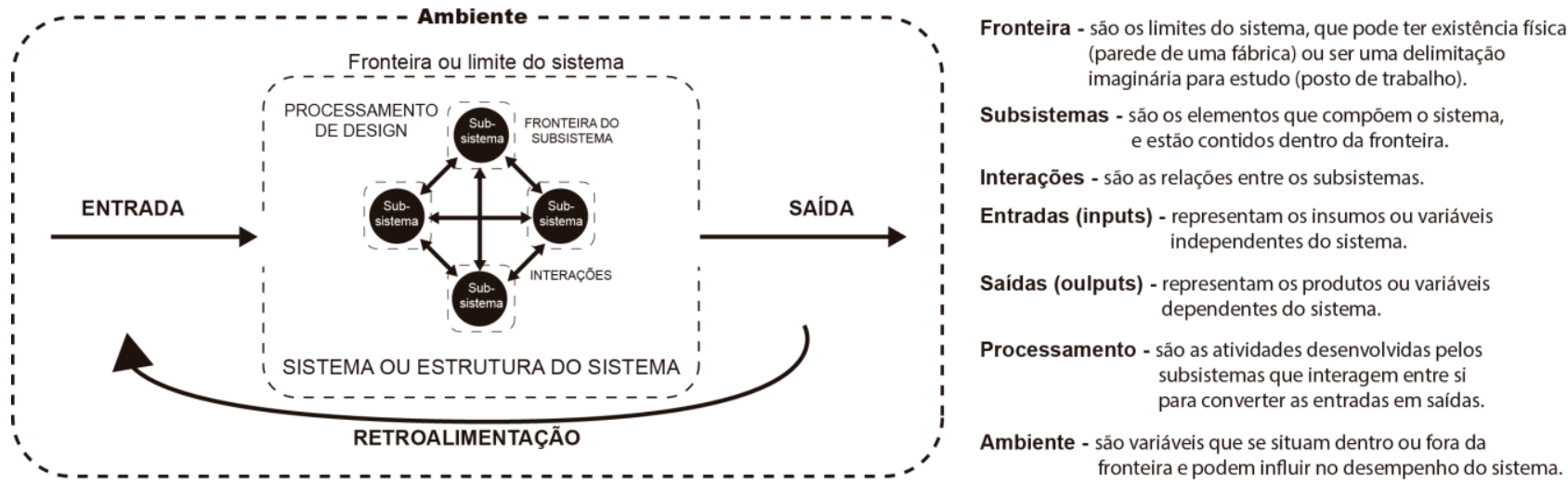

Ambiente - săo variáveis que se situam dentro ou fora da fronteira e podem influir no desempenho do sistema.

Fonte: Adaptada de Santos (2000), Vasconcellos (2013) e Aros (2016)

Tanto na entrada quanto na saída são processadas informações, e o resultado do processo de design não é o projeto do produto ou serviço e sim mais informações. Ainda possui a retroalimentação do sistema que é a volta de uma parte da saída (resultado do comportamento, do funcionamento do sistema, ou da interação do usuário com o produto ou serviço) à entrada como informação e que vai influir sobre o seu comportamento subsequente (VASCONCELLOS, 2013). Nessa interação dinâmica das partes é necessário compreender os processos isoladamente, bem como os problemas encontrados na organização e na ordem que os unifica. Assim, a AS pode ampliar a visão de Gestão de Design (GD), entendendo a organização "como um sistema aberto, em que as interferências ou problemas detectados podem ser de ordem ambiental, social ou econômica, relativos as interferências e relações internas e externas à organização e ao sistema" de GD (SILVA; FIGUEIREDO, 2010, p. 9). Por isso, segundo Silva e Figueiredo (2012), as teorias de design, de GD e de Abordagem Sistêmica de Design, quando aliadas, ajudam a entender como é ter uma perspectiva sistêmica do contexto (SILVA; FIGUEIREDO, 2012). A próxima seção aborda o Design de Serviço.

\section{Design de Serviço}

Inserido neste contexto, encontra-se o Design de Serviço que ajuda a inovar, criar novos, ou aperfeiçoar serviços, tornando-os mais úteis, usáveis e desejáveis para os clientes, e eficiente bem como eficaz para as organizações. É um novo campo holístico, multidisciplinar e integrativo, por conectar as áreas de marketing, design, gestão de empresa e pesquisa de cliente (MORITZ, 2005). Segundo Bohren et al. (2015, p.5), o Design de Serviço "aplica métodos centrados no usuário para inovar e dar forma aos serviços organizados em torno das necessidades e desejos das pessoas, levando em consideração as restrições e as possibilidades dos provedores de serviços e ambientes". Complementando essa informação, Stickdorn e Scheneider (2011) destacam cinco princípios para o Design de Serviço que são: centrado no usuário, co-criativo, sequencial, evidenciado e holístico.

Para Kotler (2005), o serviço é qualquer ato ou desempenho intangível que uma parte possa oferecer a outra sem resultar na propriedade de nada, mas que pode ou não estar ligada a um aspecto tangível (produto físico). Dentre os processos metodológicos utilizados no Design de Serviço está o Design Thinking de Serviços que possui um conjunto de ferramentas com "uma variedade de técnicas para desenvolver uma compreensão aprofundada dos clientes e suas necessidades" (RAU; ZBIEK; JONAS, 2017, p. 49, tradução do autor). Além disso, os autores 
relatam que o processo de Design de Serviço: "se concentra no usuário; promove uma visão sem restrições do problema; reconhece a necessidade de uma perspectiva colaborativa e sistêmica; e enfatiza a importância das visualizações no processo de design" (RAU; ZBIEK; JONAS, 2017, p. 49, tradução do autor). Entretanto, as ferramentas e os métodos de Design Thinking de Serviço são selecionados e adaptados para atender aos requisitos de desenvolvimento de serviços. A próxima seção relata sobre a gamificação que pode ser mais uma dessas ferramentas ou métodos utilizados no Design de Serviço.

\section{Gamificação}

Paralelamente à evolução da tecnologia, surgem novas ferramentas e métodos que auxiliam as pessoas a solucionarem problemas como o engajamento empresarial ou a colaboração durante o processo criativo de um serviço. De acordo com Formanski (2016), transversalmente a esse avanço, surge o conceito de gamificação (que deriva do termo original em inglês gamification) originado na área de Design de jogo, que é uma atividade que cria e define estruturas e regras de jogo visando à geração de experiências significativas.

A gamificação vem sendo compreendida como a aplicação de elementos, mecanismos, dinâmicas e técnicas de jogos no contexto fora do jogo com o "objetivo de resolver problemas práticos ou de despertar engajamento entre um público específico", não sendo necessariamente desenvolver um game (COHEN, 2011; VIANNA et al., 2013, p.13; NAVARRO, 2013). Os elementos, mecânicas, dinâmicas e técnicas incluem o lançamento de desafios, cumprimento de regras, metas claras e bem definidas, efeito surpresa, linearidade dos acontecimentos, conquista por pontos e troféus, estatísticas e gráficos com o acompanhamento da performance, superação de níveis e criação de avatares (personificação imaginária da própria pessoa e do seu estado de espírito na forma de um personagem). Já o contexto fora do jogo relaciona-se com situações pertencentes à realidade do dia a dia profissional, escolar e social do indivíduo (NAVARRO, 2013; VIANNA et al., 2013). O processo de implementação da gamificação consiste em: compreender o problema e o contexto; definir problemas a resolver, metodologias e mecânicas do jogo; implementar e testar.

A gamificação funciona como uma ferramenta a disposição do Design que pode simplificar um trabalho ou melhorar o processo dos serviços, e influenciar ou modificar positivamente os comportamentos humanos. Assim, a motivação é uma das grandes fontes exploradas pela gamificação. O termo motivação vem da palavra "motivus" em latim e significa o "ato ou efeito de movimentar", ou seja, estar ou ser motivado é ser movido a fazer algo (WERBACH; HUNTER, 2012). "A gamificação não é simplesmente o jogo a ser criado, mas sim o método de aplicação de ideias, o processo em si, seja ele de aprendizagem ou não" (DALLAGNOL, 2016, p.37). Ainda, segundo o autor, esta ferramenta pode ser utilizada no processo de Gestão de Design (GD) buscando atingir um novo método de atender, motivar e até influenciar o usuário em suas decisões, de modo que ele aproveite o conteúdo disponibilizado por meio de estratégias utilizadas pelo jogo.

Dessa forma, a gamificação pode fornecer experiências atraentes ao promover aprendizagem e engajamento entre os participantes. Conforme Dorling e Mccaffery (2012), o engajamento ocorre quando o cérebro é premiado e evoca emoções positivas em uma pessoa. Tendo isso em vista, percebe-se que as oportunidades de aplicação da gamificação em empresas vão desde ter clientes mais engajados até permitir a inovação (ERDÕS; KALLÓS, 2014). Hu (2003) apresenta a inovação como um processo de produção de conhecimento, exigindo criatividade e envolvendo a incerteza com 
relação aos resultados. E um dos quesitos para que haja inovação é o engajamento dos colaboradores que implica em ter pessoas mobilizadas em prol da realização de uma causa ou a um objetivo específico (DUTRA, 2013).

"A gamificação entra no contexto como a inovação a ser utilizada dentro da gestão, trazendo por meio de jogos, a integração necessária para a solução do problema relatado pela empresa ou instituição em questão, atuando como facilitador entre setores." (DALLAGNOL, 2016, p. 67). O autor propõe que a GD utilize a gamificação para suprir as necessidades, como a troca de conhecimento e informações, tanto de empregado para empregado, quanto de empregado para administrador, captando insatisfações e melhorias que podem ser implementadas, bem como os demais dados nos demasiados setores que a empresa possui.

Dessa forma, o principal objetivo da gamificação, inserida no processo de GD como ferramenta, é viabilizar uma nova forma de experiência ao praticar o já vivenciado, induzir o estudante, trabalhador ou usuário a ver de maneira diferenciada e divertida as tarefas que deve realizar. A próxima seção apresenta as etapas metodológicas.

\section{Procedimentos Metodológicos}

A pesquisa é caracterizada por sua natureza como pesquisa básica e teórica, pela abordagem como quantitativa (devido ao levantamento bibliométrico) e qualitativa (devido à revisão bibliográfica), pelos objetivos como exploratório por buscar compreender como a gamificação pode ser aplicada no Design de Serviços e identificar se já existem métodos para isso. De acordo com os procedimentos metodológicos a pesquisa é classificada como levantamento bibliográfico de dados secundários, por utilizar pesquisas científicas publicadas em bases de dados eletrônicos e livros. (GIL, 2010; VIRGILLITO, 2010; CRESWELL, 2016).

Este artigo é o resultado da junção de duas Revisões Sistemáticas da Literatura (RSL). A primeira, sobre gamificação, criatividade e Design de Serviço, foi realizada em setembro de 2017 no repositório da UFSC com a utilização do método denominado Canvas para Bibliometria (Figuras 2 e 3) proposto por Medeiros et al. (2015).

Figura 2 - Revisão Sistemática com o Método Canvas para Bibliometria

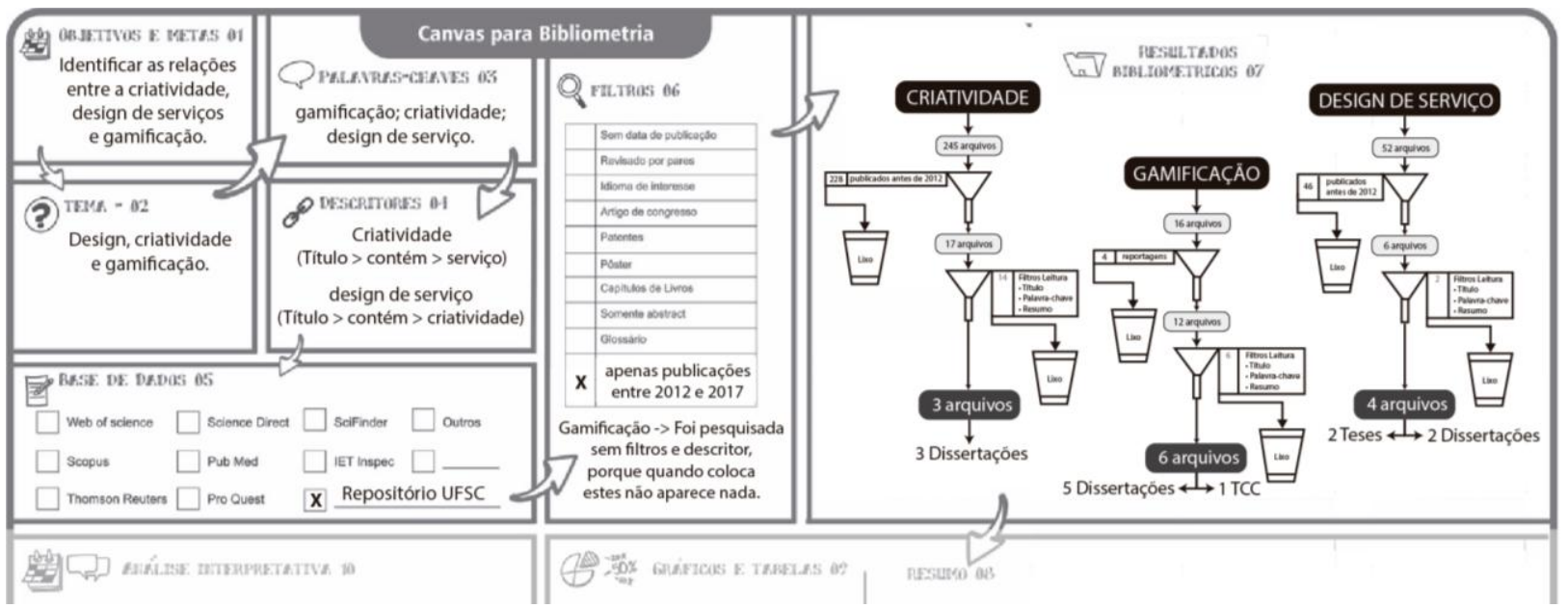

Fonte: Elaborado pelos autores com base em Medeiros et al. (2015) 
Figura 3 - Bibliometria da Revisão Sistemática da Gamificação

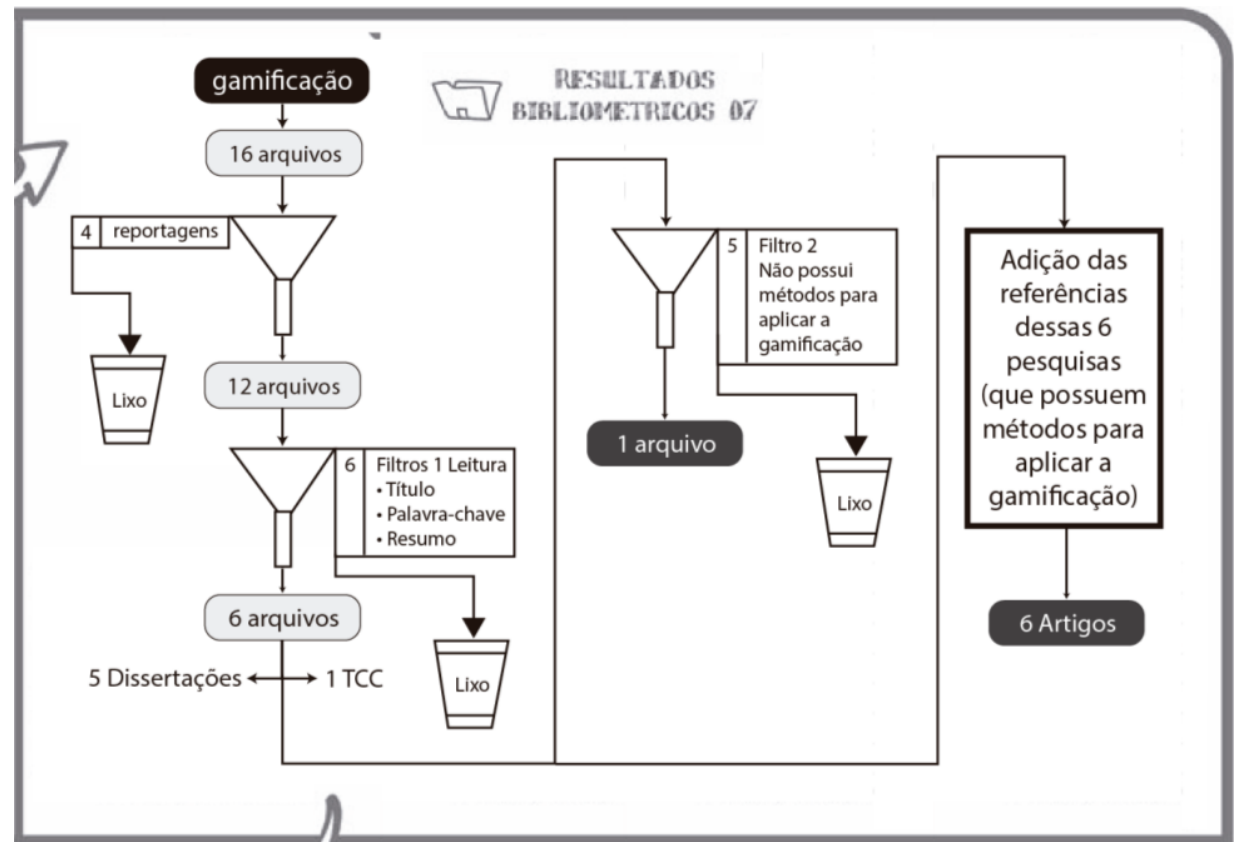

Fonte: Elaborado pelos autores com base em Medeiros et al. (2015)

Para esta pesquisa será considerado apenas o material coletado com o descritor "gamificação". Destas pesquisas, apenas uma foi incluída no portfólio final, além disso, seis pesquisas presentes nas referências bibliográficas dos trabalhos encontrados foram incluídas no portfólio final.

A segunda trata-se de uma RSL de caráter exploratório que visa revisar as pesquisas científicas realizadas sobre o processo de prototipagem de serviços por meio da gamificação, com vistas à Gestão de Design por uma Abordagem Sistêmica. O principal questionamento da RSL foi identificar quais pesquisas foram realizadas no processo de Design de Serviços que tinham como intuito a aplicação da gamificação, com vistas à Gestão de Design por uma Abordagem Sistêmica. E um dos questionamentos secundários foi identificar os métodos gamificados que vêm sendo utilizados no Design de Serviços. As buscas foram realizadas nas bases Scopus, Scielo e Web of Science (periódicos), CAPES (teses e dissertações) e ProQuest (periódicos, teses e dissertações), no período de 24 de outubro a 14 de novembro de 2017, com a string apresentada na Figura 4. Foram buscados artigos, teses, dissertações, capítulos de livros e livros que estejam dispostos nas áreas de ciências sociais ou ciências sociais aplicadas, e escritos em português e inglês.

Figura 4 - Resultado das buscas

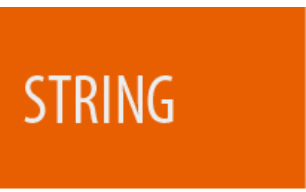

("prototyp*" OR "model*") AND ("service* design*" OR "service* develop*"

OR "service* innovation*") AND (("gamific*" OR "learn*" OR "simulat*"

OR "experienc*") OR ("gamific*") OR (manag* OR strateg*) OR (("gamific*"

OR "learn*" OR "simulat*" OR "experienc*") AND (manag* OR strateg*)))
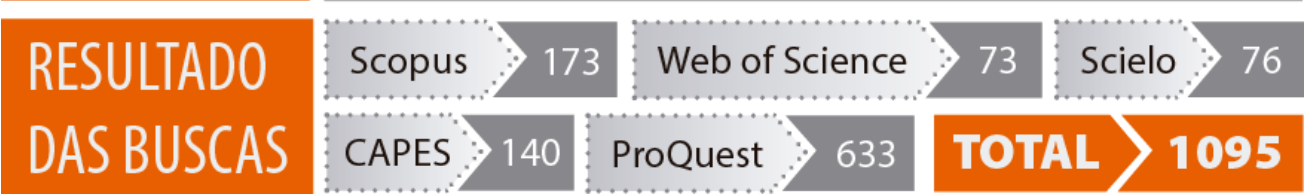

Fonte: Elaborado pelos autores 
A Figura 5 apresenta o processo executado na Revisão Sistemática da Literatura (RSL) que foi esquematizado de forma sintetizada para facilitar a compreensão.

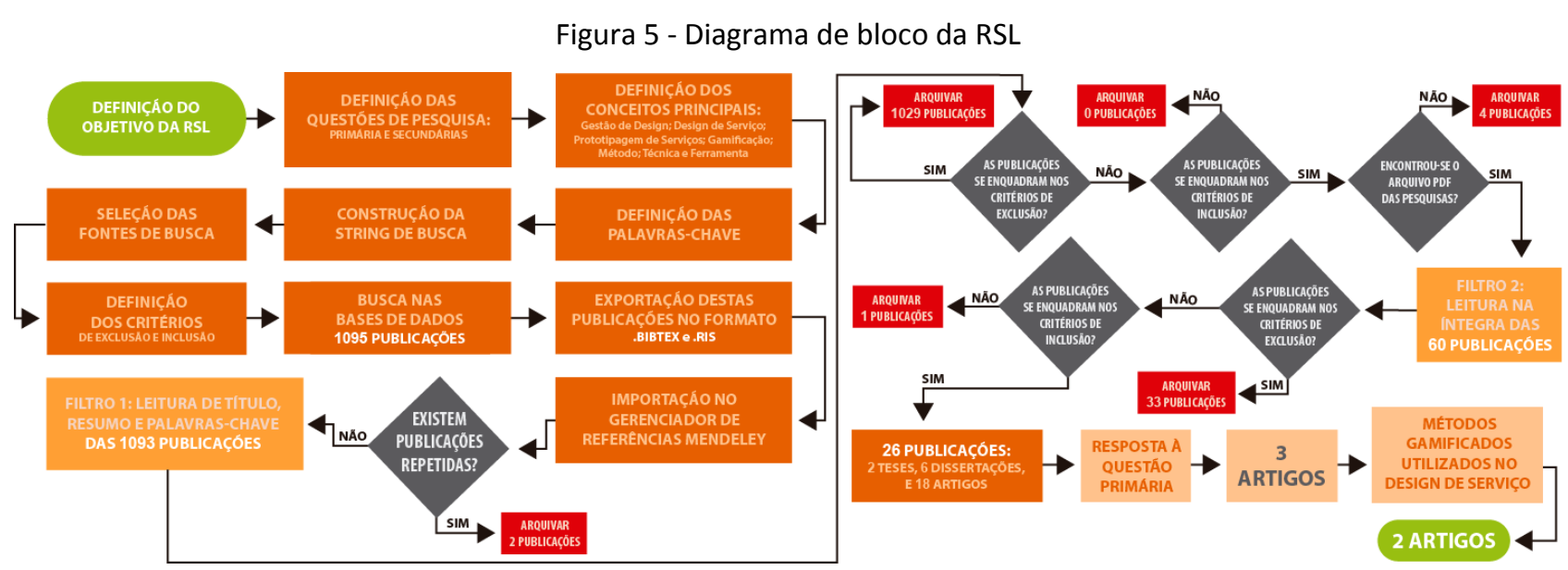

Fonte: Adaptada de Santos (2000), Vasconcellos (2013) e Aros (2016)

As pesquisas utilizadas na análise são mencionadas na próxima seção em um gráfico que mostra a classificação destas quanto ao tipo de referência (Artigo, Site, Livro, Dissertação). Os dados encontrados na pesquisa foram apresentados em um diagrama que exibe os métodos de gamificação com os respectivos autores e ano. A próxima seção apresenta os resultados e a discussão destes.

\section{Resultados}

Essa seção se divide em dois itens: a primeira composta por um gráfico que lista as pesquisas utilizadas para a análise e um diagrama que apresenta os métodos de gamificação; e na sequência relatam-se a discussão sobre os métodos que as empresas prestadoras de serviços utilizaram para aplicar a gamificação em suas atividades.

\subsection{Portfólio utilizado para a análise}

As Figuras 6 e 7 apresentam as pesquisas utilizadas na análise e a extração dos métodos apresentados na subseção 7.2.

Figura 6 - Portfólio Final das pesquisas encontradas nas RSL's

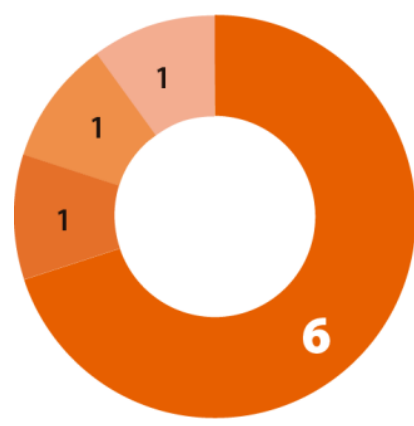

\begin{tabular}{|c|c|c|c|}
\hline $\begin{array}{l}\text { 1. Kappen (2013); } \\
\text { 2. Neeli (2012); }\end{array}$ & \multicolumn{3}{|c|}{$\begin{array}{l}\text { 3. Vieira et al. (2012); } \\
\text { 4. Liu, Alexandrova e Nakajima (2011) }\end{array}$} \\
\hline \multicolumn{4}{|c|}{ Artigos (RSL de outubro à novembro de 2017) } \\
\hline 1. Patrício (2016); & \multicolumn{2}{|c|}{ 2. Klapztein e Cipolla (2016) } & \\
\hline \multicolumn{2}{|c|}{$\begin{array}{l}\text { Site de Empresa (RSL de } \\
\text { setembro de } 2017-\text { RB) }\end{array}$} & $\begin{array}{l}\text { Livro (RSL de setembro } \\
\text { de } 2017 \text { - RB) }\end{array}$ & $\begin{array}{l}\text { Dissertaçăo (RSL de } \\
\text { setembro de } 2017 \text { - RB) }\end{array}$ \\
\hline \multicolumn{2}{|c|}{ 1. Game On! Lab (2015) } & 1. Chou (2015) & 1. Zotti (2014) \\
\hline
\end{tabular}

Fonte: Elaborado pelos autores 


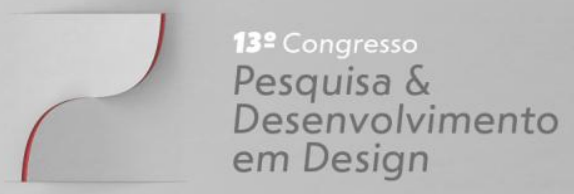

Figura 7 - Diagrama composto pelos Métodos de Gamificação

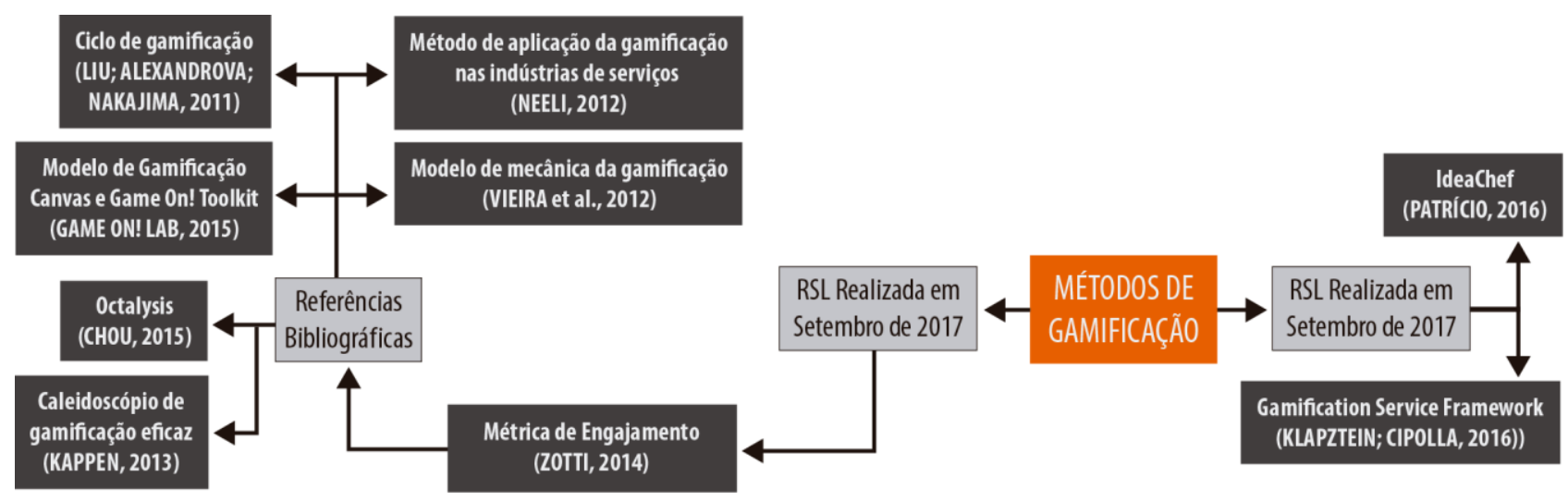

Fonte: Elaborado com base nos autores citados

A partir destas RSL's, apresenta-se na próxima seção a descrição dos métodos encontrados nessas pesquisas que podem ser utilizados para aplicar a gamificação no Design de Serviço.

\subsection{Discussão dos Resultados: métodos para aplicar a Gamificação}

Segundo Dallagnol (2016), já existem diferentes métodos para se obter o aproveitamento da gamificação no setor em que almeja-se colaboração. Estes são oferecidos e aplicados de forma distinta dependendo do público que o utilizará e dos dados que se pretende obter. Dessa forma, encontraramse, nas RSL's realizadas, dez métodos gamificados direcionados para o Design de Serviço pelo viés da Gestão de Design (GD) e Abordagem Sistêmica (AS). Estes são relatadas nos próximos parágrafos.

Zotti (2014) desenvolveu a "Métrica de Engajamento" que é baseada na inserção de mecânicas de jogos alinhadas com dinâmicas de jogos (gamificação) para motivar a participação de cidadãos e gestores públicos em processos de gestão colaborativa. $O$ autor informa que a métrica deve ser modelada de acordo com o perfil dos usuários, com os mecanismos disponíveis e com as necessidades do contexto de aplicação. Em resumo, a métrica consiste em cinco passos: 1) Analisar os elementos de gamificação; 2) Estabelecer objetivos e benefícios da gamificação; 3) Identificar os usuários e as ações; 4) Estabelecer um ranking padrão para determinar o grau de importância de cada ação; e 5) Medir o engajamento.

Neste contexto, encontrou-se a empresa Game On! Lab (2015) que possui dois métodos gamificados: o Modelo de Gamificação Canvas; e o Game On! Toolkit (Figura 8). O primeiro método, que é ágil, flexível e sistemático, foi criado por Sergio Jiménez com o intuito de ajudar a encontrar e avaliar soluções baseadas em Design de Jogo e desenvolver comportamentos em ambientes em que não haveria jogos. Já o segundo método permite que as empresas de serviços projetem seus próprios processos gamificados de forma sistemática por meio de uma tela com base no Modelo Canvas, além de incluir o acesso a vídeos que mostram como gamificar as atividades dos usuários. 
Figura 8 - Modelo de Gamificação Canvas e Game On! Toolkit
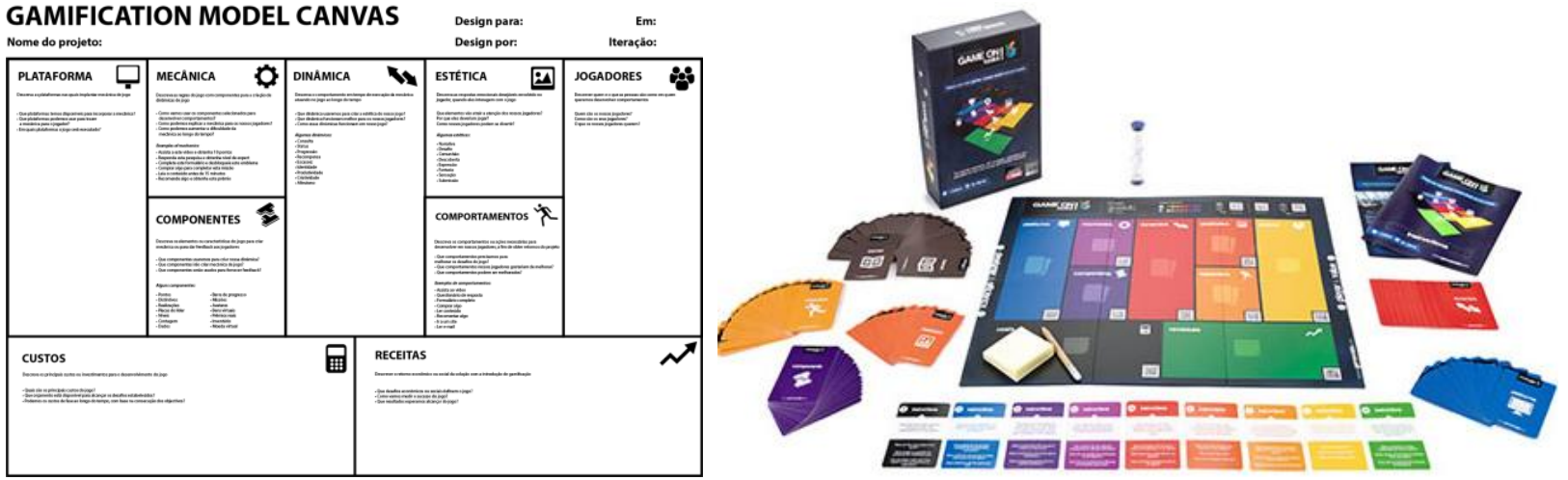

Fonte: Game On! Lab (2015)

Chou (2015) realizou uma pesquisa mais profunda no qual percebeu que quase todos os recursos do jogo de sucesso para certas unidades centrais dentro de nós nos motivam para uma variedade de decisões e atividades. Notou, também, que diferentes tipos de jogos e técnicas nos impulsionam de forma diferente, alguns por meio da inspiração e capacitação, e outros por meio da manipulação e obsessão. O resultado final de sua pesquisa gerou o método Octalysis (quadro de design gamification, vide Figura 9) que é composto por oito unidades centrais: Significado; Empoderamento; Influência social; Imprevisibilidade; Prevenção; Escassez; Propriedade; e Realização. Para o autor tudo o que uma pessoa faz é baseado em uma ou mais dessas unidades (inclusive os produtos, serviços ou sistemas), e quando não há nenhuma dessas atividades atrás de uma ação desejada há motivação zero e nenhuma ação ocorre.

Figura 9 - Octalysis (quadro de design gamification)
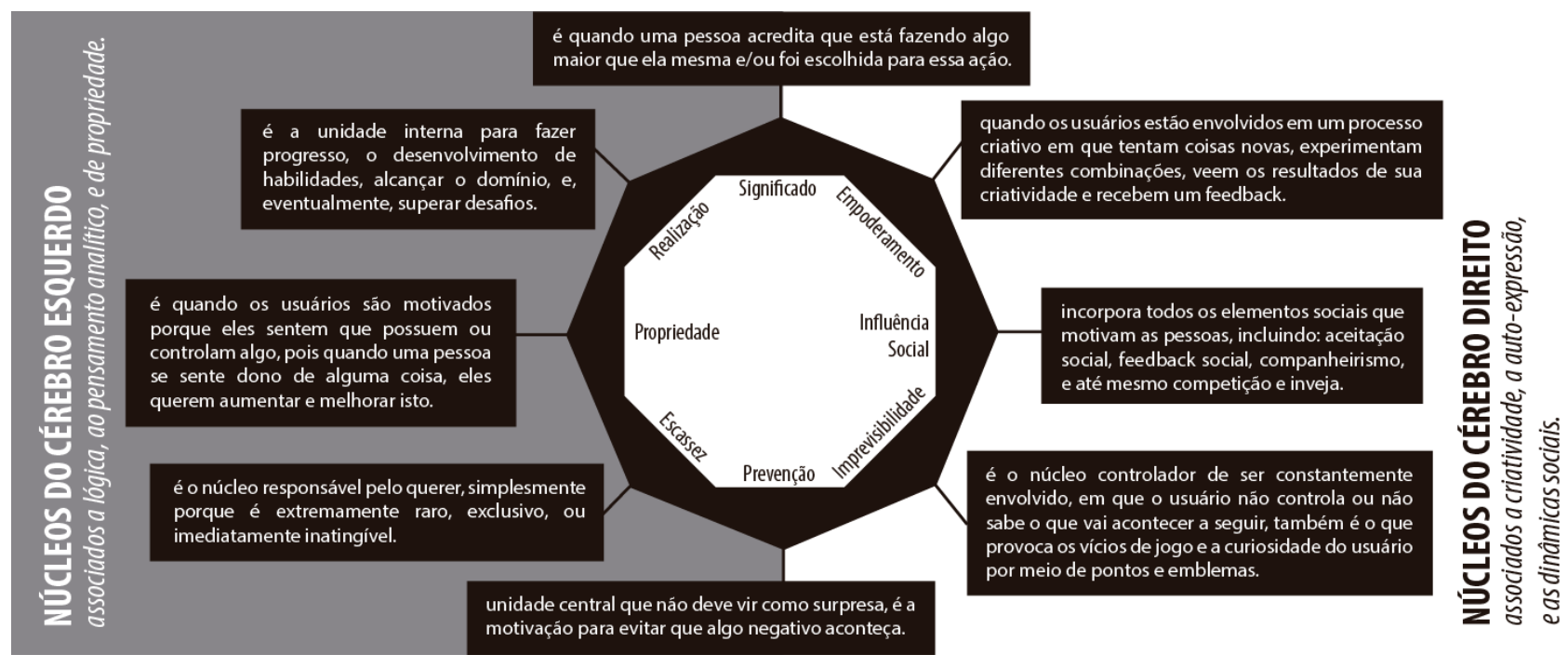

Fonte: Adaptado de Chou (2015)

Com base nos modelos atuais do projeto de jogo, da teoria da autodeterminação e dos princípios do projeto dos sistemas, Kappen (2013) propôs um modelo centrado no design e na gamificação denominado como o caleidoscópio da gamificação eficaz. As camadas deste modelo 
fornecem diretrizes de design para a aplicação eficaz da gamificação em projetos empresariais. A Figura 10 ilustra a complexidade das relações de cada anel com os anéis adjacentes em uma associação de exibição superior.

Figura 10 - Caleidoscópio da Gamificação Eficaz

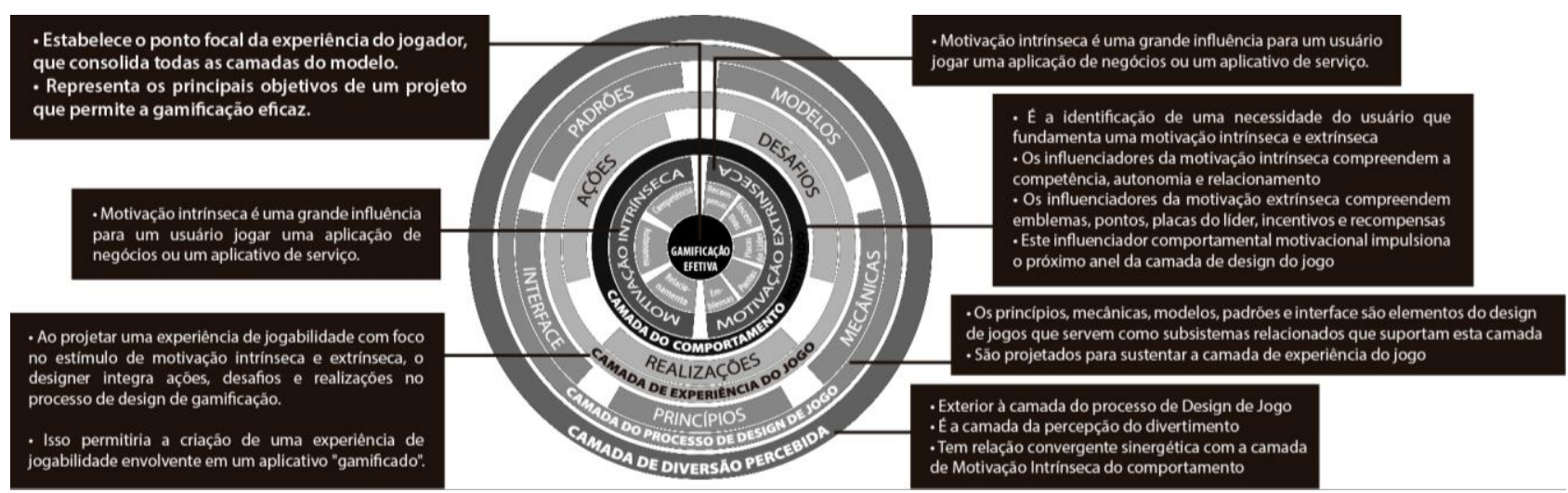

Fonte: Kappen (2013)

Diferentemente dos demais autores, Neeli (2012) aplicou o "Método de aplicação da gamificação" na indústria de Business Process Outsourcing (BPO) para aumentar o engajamento do funcionário e, assim, gerenciar os desafios relacionados com o empregado encontrado em BPO ou qualquer indústria de serviços. Já Vieira et al. (2012) elaboraram o "modelo de mecânica de jogo" (Figura 11) que foi construído com ênfase na narrativa (estória), no qual as mecânicas e os principais elementos relacionados são as atividades, perfil (avatar), regras e feedback.

O ciclo da gamificação (Figura 11), desenvolvido por Liu, Alexandrova e Nakajima (2011), foi construído com base no sistema de pontos (contagem, moeda virtual, ponto da experiência, etc.) por ser uma resposta rápida para promover a participação dos indivíduos. A proposta deste método é criar um ciclo de gamificação para sistemas fora do contexto de jogo em que a interação começa com um objetivo ou desafio. Cada vez que o usuário alcança um objetivo pequeno são fornecidas algumas recompensas (suportadas pelo sistema de pontos), placar dos líderes (global ou parcialmente) e emblemas aos jogadores para motivar a competitividade, o que eventualmente resulta em mudança de status do jogador virtual em sua rede social ou sistema.

Figura 11 - Modelo de mecânica da gamificação (à esquerda) e Ciclo da gamificação (à direita)
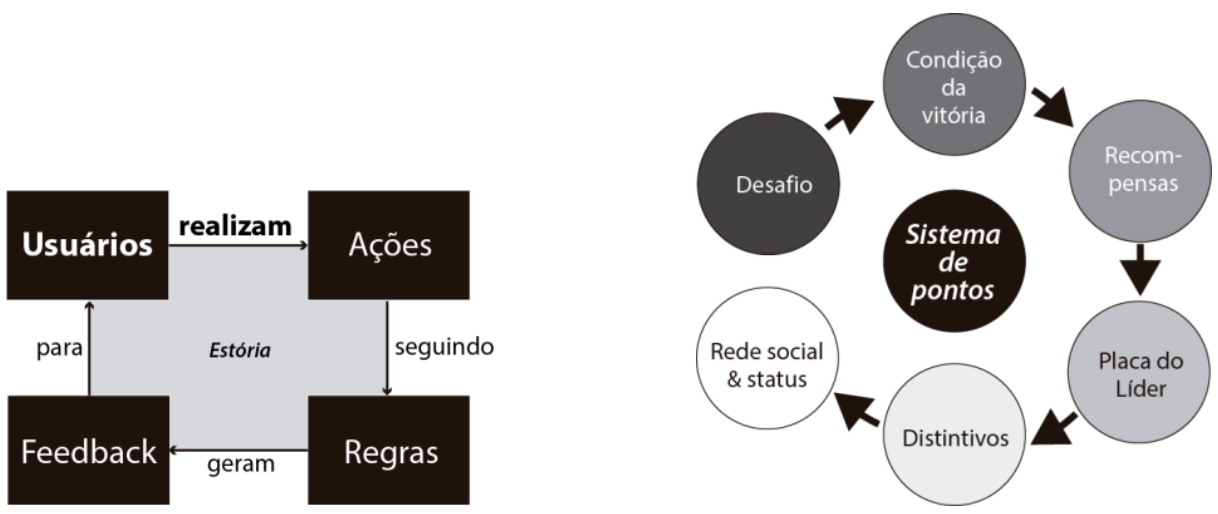

Fonte: Vieira et al. (2012, à esquerda) e Liu, Alexandrova e Nakajima (2011, à direita) 
O IdeaChef (jogo de tabuleiro com metáforas de culinária, apresentado na Figura 12) integra conceitos de Design de Serviços com técnicas de resolução de problemas e inovação empresarial, que abordam um desafio, necessidade ou problema específico, relacionado aos processos internos ou ao mercado externo. Este visa converter ideias de alto potencial em conceitos de trabalho ou protótipos. (PATRícIO, 2016).

Figura 12 - IdeaChef
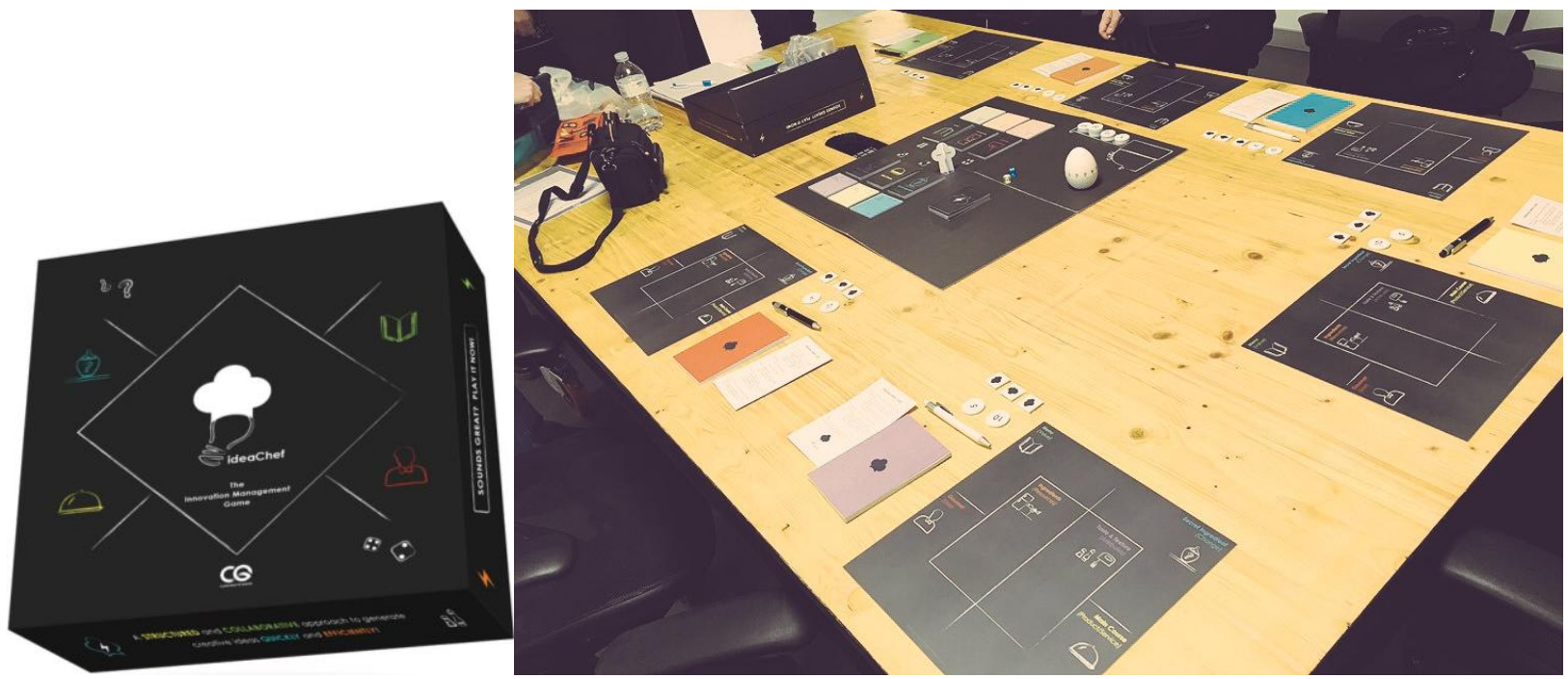

Fonte: Patrício (2016)

O Gamification Service Framework (GSF, Figura 13) combina o método de Pesquisa-Ação em Design, Design de Jogo (Gamificação) e Design de Serviço (Jornada do Serviço) para gerenciar serviços de forma simples (KLAPZTEIN; CIPOLLA, 2016).

Figura 13 - Estruturação do GSF (à direita) e Modelo de aplicação do GSF (à esquerda)

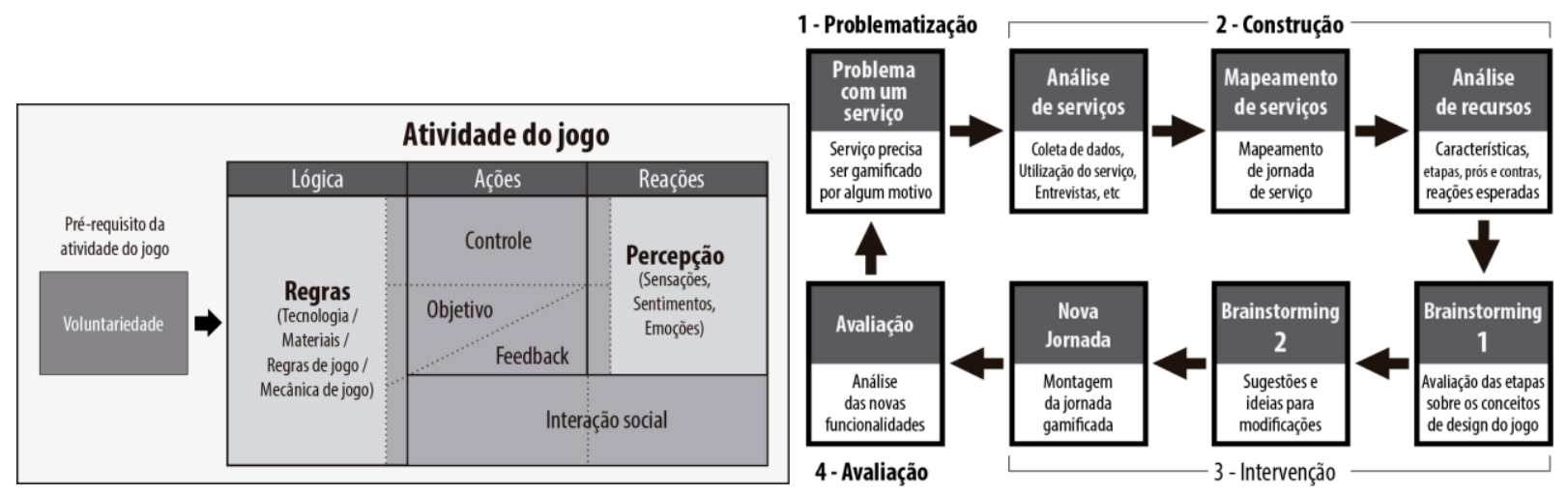

Fonte: Klapztein e Cipolla (2016)

Infere-se, com base nos autores citados na fundamentação teórica e naqueles encontrados nas RSL's, que a aplicação dos métodos gamificados no Design de Serviço pode auxiliar na colaboração, engajamento e motivação da equipe de trabalho e dos usuários. 


\section{Considerações Finais}

Diante do exposto nas seções anteriores, conclui-se que a gamificação pode ser aplicada no Design de Serviço por meio de métodos gamificados cujo seus processos incluem mecânicas, técnicas, dinâmicas e elementos de jogos. Ressalta-se ainda que, apesar de poucos, já existem métodos gamificados que podem ser utilizadas para aplicar a gamificação no Design de Serviço (mesmo que alguns não sejam específicos para isso).

Deste modo, por meio das RSL's, foram identificados dez tipos de métodos, que podem auxiliar a aplicação da gamificação no processo de Design de Serviço pelo viés da Gestão de Design e Abordagem sistêmica, que são: a Métrica de Engajamento (ZOTTI, 2014); o Modelo de Gamificação Canvas e o Game On! Toolkit (GAME ON! LAB, 2015); o Octalysis (CHOU, 2015); o Caleidoscópio de gamificação eficaz (KAPPEN, 2013); o Método de aplicação da gamificação nas indústrias de serviços (NEELI, 2012); o Modelo de mecânica da gamificação (VIEIRA et al., 2012); o Ciclo de gamificação (LIU; ALEXANDROVA; NAKAJIMA, 2011); o IdeaChef (PATRÍCIO, 2016); e o Gamification Service Framework (KLAPZTEIN; CIPOLLA, 2016).

Destaca-se, com base em Dallagnol (2016), que a gamificação quando inserida no contexto de Gestão de Design (GD) confere ao designer um caráter baseado em planejamento e em busca de resultados. Isso faz com que as atividades do gestor de design sejam direcionadas para a criatividade, auxiliando a promoção de ideias que proporcionam resultados duradouros, por estarem baseadas em ações práticas e integradas, em que os envolvidos pela gamificação se sentem motivados a contribuir e, por isso, conseguem atingir os objetivos desejados pelas organizações. Por estes motivos, a gamificação pode potencializar a criatividade por meio da motivação e do engajamento, estes ajudam na resolução de problemas no Design de Seviço e na GD.

Como limitação de pesquisa, ressalta-se a dificuldade de encontrar um método específico para a aplicação da gamificação no Design de Serviço, o que torna-se uma oportunidade para pesquisa: o desenvolvimento de um método gamificado específico para o processo de Design de Serviço, pelo viés da Gestão de Design e Abordagem Sistêmica.

\section{Referências}

AGUIAR, M. C.; MERINO, E. A. D.; MERINO, G. S. A. D.; TRISKA, R. Gestão de design e sua contribuição para organizações no setor do artesanato: proposição e implementação de um plano estratégico para a ARA Associação Ribeirão de Artesanato. In: IDEMI, 4., 2015, Florianópolis. Anais da Fourth International Conference on Integration of Design, Engineering and Management for innovation. Florianópolis: UDESC, 2015.

AROS, K. C. Elicitação do processo projetual do Núcleo de Abordagem Sistêmica do Design da Universidade Federal de Santa Catarina. Dissertação (mestrado) - Centro de Comunicação e Expressão, Programa de Pós-Graduação em Design, Universidade Federal de Santa Catarina, Florianópolis, SC, 2016.

BERTALANFFY, L V. Teoriageral dos sistemas: fundamentos, desenvolvimento e aplicações. 8. ed. Petrópolis, RV: Vozes, 2014.

BEST, K. Fundamentos de gestão do design. Porto Alegre: Bookman, 2012.

BOHREN, M. A. et al. Formative research and development of innovative tools for "Better Outcomes in Labour Difficulty" (BOLD): study protocol. Reproductive Health, v. 12, 2015.

CAPRA, F. A teia da vida: uma nova compreensão científica dos sistemas vivos. 12. ed. São Paulo: Cultrix, 2010. 
CASTRO, F. S.; FERNANDES, A. M. da R. Aprendendo química orgânica através de gamificação. Anais do Computer on the Beach, 2013, p. 328-330.

CHOU, Y. K. Actionable Gamification: Beyond Points, Badges, and Leaderboards. Editora Leanpub, 2015. CRESWELL, J. W. Projeto de pesquisa: métodos qualitativo, quantitativo e misto. Porto Alegre: SAGE, 2010. COHEN, A. M. The gamification of education. Futurist, v. 45, n. 5, p. 16-17, 2011.

DALLAGNOL, V. A inserção da gamificação no processo de gestão de design. Dissertação (mestrado) - Centro de Comunicação e Expressão, Programa de Pós-Graduação em Design, Universidade Federal de Santa Catarina, Florianópolis, 2016.

DALE, S. Gamification: making work fun, or making fun of work? Business Information Review, p. 82-90, jun. 2014. DORLING, A.; MCCAFFERY, F. The Gamification of SPICE. Software Process Improvement and Capability Determination Communications in Computer and Information Science, v. 290, 2012.

DUTRA, J. A inovação empresarial depende do engajamento das pessoas. 2013. Disponível em: <http://www.senior.com.br/a-inovacao-empresarial-depende-do-engajamento-daspessoas/\#sthash.ZTT4MnVw.LNRAwnQa.dpuf>. Acesso em: 26 ago. 2017.

ERDÕS, Erenc; KALLÓS, Gábor. Benefit Evaluation Model for Gamified Add- Ons in Business Software. Acta Polytechnica Hungarica, p. 109-124, 2014.

FRANCISCO, P. H. D. Avaliação estética de protótipos no design de serviço. 2016. Dissertação (Mestrado em Design) - Setor de Artes, Comunicação e Design da Universidade Federal do Paraná, Curitiba, 2016.

FORMANSKI, F. N. Aplicabilidade da gamificação no contexto empresarial. Dissertação (mestrado) - Centro Tecnológico, Programa de Pós-Graduação em Engenharia e Gestão do Conhecimento, Universidade Federal de Santa Catarina, Florianópolis, 2016.

GAME ON! LAB. Gamification Model Canvas. 2015. Disponível em: <http://www. gameonlab.com/canvas/>. Acesso em: 27 fev. 2018.

.Toolkit. 2015. Disponível em: <http://www.gameonlab.com/toolkit/>. Acesso em: 27 fev. 2018.

GIL, A. C. Como elaborar projetos de pesquisa. São Paulo: Atlas, 2010.

HU, A. R\&D Organization, monitoring intensity and innovation performance in Chinese industry. Econ. Innov. New Techn., v. 12, 2003.

KAPPEN, Dennis L.; NACKE, Lennart E. The kaleidoscope of effective gamification: deconstructing gamification in business applications. Proceedings of the First International Conference on Gameful Design, Research, and Application, 2013, p. 119-122.

KLAPZTEIN, S.; CIPOLLA, C. From Game Design to Service Design. Simulation \& Gaming, v. 47, n. 5, p. 566-598, 2016.

KOTLER, P. Administração de Marketing. São Paulo: Prentice Hall, 2005.

KRUCKEN, L. Design e território: valorização de identidades e produtos locais. 1. ed. v. 1. São Paulo: Nobel, 2009. LIU, Y.; ALEXANDROVA, T.; NAKAJIMA, T. Gamifying Intelligent Environments. Proceedings of the 2011 international ACM workshop on Ubiquitous meta user interfaces, p. 7-12, 2011.

MCGONIGAL, J. Realidade em jogo: por que os games nos tornam melhores e como eles podem mudar o mundo. Rio de Janeiro: Best Seller, 2012. 
MEDEIROS, I. L. de et al. Revisão Sistemática e Bibliometria facilitadas por um Canvas para visualização de informação. Revista Brasileira de Design da Informação, v. 12, n. 1, 2015, São Paulo, p. 93-110.

MORIN, E. O método 1: a natureza da natureza. Porto Alegre: Sulina, 2005.

MORITZ, S. Service Design: practical access to an evolving fiel. London: Köln International School of Design, 2005.

MOZOTA, B. B. de. Gestão do design: usando o design para construir o valor de marca e inovação corporativa. Porto Alegre: Bookman, 2011.

NAVARRO, G. Gamificação: a transformação do conceito do termo jogo no contexto da pósmodernidade. 2013. Disponível em: <http://200.144.182.130/celacc/sites/default/files/media/tcc/ 578-1589-1-PB.pdf>. Acesso em: 26 ago. 2017.

NEELI, B. K. A Method to Engage Employees using Gamification in BPO Industry. Third International Conference on Services in Emerging Markets, p.142-146, 2012.

OLENSKI, Steve. How to Solve with Gamification. 2014. Disponível em: <http://www.forbes.com/sites/steveolenski/2014/03/05/how-to-solve-your-biggest-marketingproblems-with-gamification/>. Acesso em: 28 out. 2017.

PATRÍCIO, R. IdeaChef ${ }^{\oplus}$ : A gamified approach for engaging teams in corporate innovation \& entrepreneurship. ISPIM Conference Proceedings. Anais...Manchester: The International Society for Professional Innovation Management (ISPIM), 2016.

RAU, C.; ZBIEK, A.; JONAS, J.M. Creating Competitive Advantage from Services: a Design Thinking Case Study from the Commodities Industry. Research Technology Management, Arlington, v. 60, n. 3, p. 48-56, May 2017.

SANTOS, F. A. dos. 0 design como diferencial competitivo: o processo de design desenvolvido sob o enfoque da qualidade e da gestão estratégica. 2. ed. Itajaí: Editora da Univali, 2000.

SILVA, C. S. ; FIGUEIREDO, L. F. Abordagem Sistêmica da Gestão de Design em Microempresas e Empresas de Pequeno Porte (MPEs). In: $9^{\circ}$ P\&D Design - Congresso Brasileiro de Pesquisa e Desenvolvimento em Design, 2010, São Paulo. P\&D Design 2010, 2010.

SILVA, C. S.; FIGUEIREDO, L. F. G. Abordagem Sistêmica em MPEs: design de sistemas para elaboração de ferramentas de gestão de design. In: 10 P\&D Design - Congresso Brasileiro de Pesquisa e Desenvolvimento em Design, 2012, São Luiz. P\&D Design 2012. São Luiz: Anais P\&D Design 2012, 2012.

STICKDORN, M.; SCHENEIDER, J. This is service design thinking: basics, tools, cases. Amsterdan: Wiley, 2012.

VASCONCELLOS, M. J. E. Pensamento sistêmico: o novo paradigma da ciência. 10. ed. Campinas, SP: Papirus, 2013.

VIANNA, Y. et al. Gamification, Inc: como reinventar empresas a partir de jogos. Rio de Janeiro: Mjv Press, 164 pp., 2013.

VIEIRA V. et al. An Exploratory Study on the Use of Collaborative Riding based on Gamification as a Support to Public Transportation. Brazilian Symposium on Collaborative Systems, 2012.

VIRGILLITO, S. B. Pesquisa de marketing: uma abordagem quantitativa e qualitativa. São Paulo: Saraiva, 2010.

WERBACH, K.; HUNTER, D. For the Win: How Game Thinking Can Revolutionize Your Business. Wharton Digital Press, 2012.

ZOTTI, A. I. Engajamento de gestores públicos e cidadãos através de uma métrica baseada em elementos de gamificação. Dissertação (mestrado) - Centro Tecnológico, Programa de Pós-Graduação em Engenharia e Gestão do Conhecimento, Universidade Federal de Santa Catarina, Florianópolis, 2014. 